\title{
Comprehensive treatment of critical coronavirus disease 2019: a single- center retrospective case series from Wuhan
}

\author{
Ke Huang ( $\nabla$ huangke_zryy@163.com ) \\ Yi Zhang \\ China-Japan Friendship Hospital

\section{Min Li} \\ China-Japan Friendship Hospital \\ Yingying Feng \\ China-Japan Friendship Hospital \\ Xin Yu \\ China-Japan Friendship Hospital \\ Ying Cai \\ China-Japan Friendship Hospital \\ Jingen Xia \\ China-Japan Friendship Hospital \\ Jun Duan \\ China-Japan Friendship Hospital \\ Qingyuan Zhan \\ China-Japan Friendship Hospital
}

China-Japan Friendship Hospital https://orcid.org/0000-0001-8933-387X

\section{Research}

Keywords: COVID-19; respiratory support techniques; glucocorticoids; convalescent plasma

Posted Date: May 5th, 2020

DOI: https://doi.org/10.21203/rs.3.rs-25658/v1

License: (c) (i) This work is licensed under a Creative Commons Attribution 4.0 International License. Read Full License 


\section{Abstract}

Background: Coronavirus disease 2019 (COVID-19) is caused by severe acute respiratory syndrome coronavirus 2 (SARS-CoV-2) and has now spread worldwide. This study aimed to provide a reference for comprehensive treatment, personal protection, and team management of patients with critical COVID-19.

Methods: The study included critical COVID-19 cases at a single centre in Wuhan, China. We retrospectively analysed data on symptoms, laboratory tests, radiology findings, treatment, and outcomes. Air samples and environmental surface swabs in the isolation ward were tested for SARS-CoV-2.

Results: Fourteen critically ill patients (mean age 62.1 years) were treated between February 4, 2020 and April 6, 2020. Less than half had underlying diseases, including hypertension $(n=6,42.9 \%)$ and diabetes $(n=4,28.6 \%)$. Laboratory tests showed decreased lymphocyte levels and increased serum ferritin and inflammatory cytokine levels. More than half of the patients received antiviral drugs, including lopinavir/ritonavir ( $\mathrm{n}=10 ; 71.4 \%$ ) and arbidol $(n=6,42.9 \%)$. Eight patients (57.1\%) received convalescent plasma, and 12 (85.7\%) received systemic glucocorticoids. Eleven (78.6\%) received highflow nasal cannula oxygen therapy, five (35.7\%) received non-invasive positive pressure ventilation, seven (50.0\%) received invasive positive pressure ventilation, and three (21.4\%) received extracorporeal membrane oxygenation. By April 6, 2020, nine (64.3\%) patients were discharged, four remained in hospital, and one had died. All air samples tested negative for SARS-CoV-2. Of 128 environmental surface swabs, one gastric tube swab and one anal tube swab were positive for SARS-CoV-2. All oropharyngeal swabs taken from medical staff tested negative for SARS-CoV-2.

Conclusions: Individualised comprehensive treatment, appropriate personal protection, and teamwork may improve the prognosis in patients with COVID-19 who are critically ill.

\section{Introduction}

A communicable disease manifesting as fever and pneumonia emerged in December 2019 and has since spread throughout the world causing a pandemic. The pathogen has been identified as a new coronavirus that has been named severe acute respiratory syndrome coronavirus 2 (SARS-CoV2). The disease syndrome has been called coronavirus disease 2019 (COVID-19). A study that included 1099 early patients with COVID-19 of varying severity showed that $5.0 \%$ required admission to the intensive care unit (ICU) and that $1.4 \%$ died(1). Another study in China found that $61.5 \%$ of critical cases admitted to the ICU died within 28 days after admission(2). SARS-CoV-2 has also caused a high number of deaths outside of China, nearly $10 \%$ of which occurred in Italy(3). There is an urgent need to decrease the mortality rate in patients who become critically ill with COVID-19.

There are no known antiviral drugs that are effective for SARS-CoV-2. Clinicians continue to treat critical cases according to their experience of acute respiratory distress syndrome and other types of viral pneumonia. Several antiviral drugs, convalescent plasma, glucocorticoids, mechanical ventilation, and extracorporeal membrane oxygenation (ECMO) have been used but not in a coordinated manner(4). Here we report a case series from a centre treating patients with critical COVID-19 on the frontline in Wuhan, China. The aim of this report is to summarize our experience of comprehensive treatment, use of personal protection, and the value of team management.

\section{Methods}

\section{Medical environment}

Our team took charge of a 50-bed isolation ward at the Sino-French branch of Tongji Hospital, which was a designated centre for patients with severe or critical COVID-19, and started to receive patients on February 4 2020. Most of the equipment used, which included monitors, ventilators, ECMO systems, bronchoscopes, ultrasound machines, and dialysers, were sourced from the China-Japan Friendship Hospital in Beijing. Six ICU beds were set aside for patients needing invasive positive pressure ventilation (IPPV) or ECMO from February 122020 onwards.

The isolation ward was divided into a clear area, a buffer area, and a contaminated area. N95 masks, goggles, face shields, and protective suits were worn in the contaminated area. Staff removed their protective equipment in the buffer area and completed their paperwork after replacement of their N95 mask in the clear area. Additional protection with a positive pressure head cover was used during high-risk procedures, such as intubation.

Our medical team included 30 doctors, 95 nurses, one respiratory therapist, and one nosocomial infection control specialist from the China-Japan Friendship Hospital in Beijing. This team was experienced in the management of patients with critical respiratory disease and had treated more than 250 cases with ECMO during the previous 2 years. Thirteen doctors (43\%, including three attendings and six fellows) from the Department of Pulmonary and Critical Care Medicine (PCCM) took charge of the treatment and $17(18 \%)$ nurses were from the ICU. Three groups of doctors cared for the patients during the day shift for 6 hours from Monday to Sunday (one for critical cases and two for severe cases). Six groups of doctors (with at least one PCCM fellow in each group) worked the night shift, and each group worked for 6 hours every 2 days. The nurses were divided into nine groups and worked for 4 hours per shift. Two nurses (at least one from ICU) cared for each patient on ECMO. A respiratory therapist managed the equipment in the ward and assisted with airway management, mechanical ventilation, and bronchoscopy. A specialist supervised the use of personal protection and nosocomial infection control. 


\section{Study Population}

All critical COVID-19 cases were admitted to our centre between February 42020 and April 6 2020, at which time the isolation ward was closed.

Patients who died before February 12 2020, when we could not carry out intubation and mechanical ventilation, were excluded. Patients whose swab tests were positive for SARS-CoV-2 RNA and those with pneumonia and a positive serum SARS-CoV-2 IgM result were defined as confirmed cases of COVID-19. Patients who needed high-flow nasal cannula (HFNC) oxygen therapy with a fraction of inspired oxygen $\left(\mathrm{FiO}_{2}\right)>0.5$, non-invasive positive pressure ventilation (NPPV), IPPV, or ECMO were defined as critical cases. Patients could be discharged or transferred to another general ward if they had a normal temperature for 3 days, their respiratory symptoms improved, the opacities on lung computed tomography scans were resolved, and two consecutive swab or sputum SARS-CoV-2 RNA tests 24 hours apart were negative.

\section{Data Collection And Analysis}

Demographic data, symptoms, laboratory tests, treatment details, and outcomes were collected retrospectively. Air samples and environmental surface swabs were also collected to test for SARS-CoV-2 RNA. Oropharyngeal swabs of all medical staff were also tested for COVID-19 pathogens in April 8 2020 after the isolation ward shut down. Continuous variables were expressed as means and categorical variables were summarised as counts and percentages. The study was independently approved by the ethics committees of the abovementioned hospital (2020-21-K16), and informed consent was waived due to the retrospective nature and urgent need to collect data.

\section{Results}

One hundred patients with COVID-19 were admitted to our centre between February 42020 and April 6 2020. Seventeen cases were defined as critical, three of whom died before February 12 2020, when we were unable to carry out intubation and mechanical ventilation, and were excluded. Finally, 14 critical cases were included into the analysis (Table 1). These patients had a mean age of 62.1 years. Six (42.9\%) had hypertension and four (28.6\%) had diabetes. Most had fever and cough, and all had dyspnoea. The lymphocyte count was decreased and the serum ferritin level was increased. All patients have increased levels of at least three inflammatory cytokines. The mean time from onset to hospitalisation was 10.8 days and from onset to hospitalisation in our centre was 20.1 days. 
Table 1

Baseline characteristics of included critical cases

Patients, $\mathrm{n}$

14

Age, mean, years

62.1

Sex, $n(\%)$

Male

Female

Comorbidities, $\mathrm{n}(\%)$

Chronic respiratory disease

Chronic heart disease

Hypertension

Diabetes mellitus

$4(28.6)$

Cerebral vascular disease

Malignancy

Symptoms, n(\%)

Fever

Cough

Dyspnoea

14(100)

Laboratory tests

Lymphocyte count, mean, $10^{\wedge 6} / \mathrm{L}^{\mathrm{a}}$

837

Ferritin, mean, $\mu \mathrm{g} / \mathrm{L}$

1711

Elevated inflammatory cytokines*, n (\%)

14(100)

Bilateral opacity on chest CT scan, $\mathrm{n}(\%)$

14(100)

Durations from onset to hospitalisation, mean, days

10.8

Duration from onset to hospitalisation in our centre, mean, days

20.1

${ }^{a}$ An elevated inflammatory cytokine level was defined as a three-fold increase in the levels of at least three of the following: interleukin- $1 \beta$ interleukin-2R, interleukin-6, interleukin-8, interleukin-10, and tumor necrosis factor

The treatment details are shown for all cases in Table 2 and Additional file 1 . Three of the 14 patients $($ cases 8, 10, 11) had received IPPV and ECMO before they were transferred to our centre. The remaining 11 patients received HFNC initially in our ward, and four (36.4\%) were successfully treated with HFNC. Five of the remaining 11 patients received NPPV; three (60.0\%) of these patients were treated successfully with NPPV and two (40.4\%) were finally intubated. Four (36.4\%) patients received IPPV after failure of HFNC or NPPV. Five (71.4\%) of seven patients who received IPPV underwent tracheostomy, three patients (cases 8, 9, and 11) received prone position ventilation, and three (42.9\%) received venous-venous ECMO. Six (85.7\%) of the patients who received IPPV showed clinical improvement; two were discharged, one was transferred to a general ward for rehabilitation, and three were transferred to another ICU. One patient received IPPV and ECMO but died due to a carbapenem-resistant Acinetobacter baumannii bloodstream infection. 
Treatment and outcome of critical cases

\begin{tabular}{|c|c|c|c|c|c|c|c|c|c|c|c|c|c|c|}
\hline \multirow[t]{2}{*}{ Patient } & \multirow{2}{*}{$\begin{array}{l}\text { Age, } \\
\text { years }\end{array}$} & \multirow[t]{2}{*}{ Sex } & \multicolumn{5}{|c|}{ Respiratory support } & \multicolumn{2}{|c|}{ Antiviral drugs } & \multirow[t]{2}{*}{$\mathrm{CP}$} & \multirow[t]{2}{*}{$A C$} & \multirow[t]{2}{*}{ GCs } & \multirow[t]{2}{*}{ IVIG } & \multirow[t]{2}{*}{ Outcome } \\
\hline & & & HFNC & NPPV & IPPV & $\begin{array}{l}\text { WV- } \\
\text { ECMO }\end{array}$ & Tracheostomy & LPV/RTV & Arbidol & & & & & \\
\hline 1 & 60 & $\mathrm{~F}$ & + & + & + & & & + & & + & + & + & + & Discharge \\
\hline 2 & 62 & $\mathrm{~F}$ & + & + & & & & + & & + & + & + & + & Discharge \\
\hline 3 & 56 & $\mathrm{~F}$ & + & + & & & & + & & & + & + & + & Discharge \\
\hline 4 & 57 & $\mathrm{~F}$ & + & + & + & & & + & & + & + & + & + & Discharge \\
\hline 5 & 65 & M & + & & & & & + & & & & + & + & Discharge \\
\hline 6 & 72 & $\mathrm{~F}$ & + & & & & & + & & & + & & + & Discharge \\
\hline 7 & 66 & $\mathrm{~F}$ & + & & & & & & + & & + & & + & Discharge \\
\hline 8 & 62 & $M$ & & & + & + & + & & + & + & + & + & + & Death \\
\hline 9 & 70 & $\mathrm{~F}$ & + & & + & & + & & + & + & + & + & + & $\begin{array}{l}\text { Transferred } \\
\text { to another } \\
\text { ICU }\end{array}$ \\
\hline 10 & 42 & M & & & + & + & + & + & & + & + & + & + & $\begin{array}{l}\text { Transferred } \\
\text { to general } \\
\text { ward }\end{array}$ \\
\hline 11 & 42 & M & & & + & + & + & + & & + & + & + & + & $\begin{array}{l}\text { Transferred } \\
\text { to another } \\
\text { ICU }\end{array}$ \\
\hline 12 & 66 & $\mathrm{~F}$ & + & & & & & + & + & & + & + & + & Discharge \\
\hline 13 & 81 & $\mathrm{~F}$ & + & & + & & + & & + & & & & + & $\begin{array}{l}\text { Transferred } \\
\text { to another } \\
\text { ICU }\end{array}$ \\
\hline 14 & 69 & M & + & + & & & & + & + & + & + & + & + & Discharge \\
\hline \multicolumn{15}{|c|}{ Abbreviations } \\
\hline $\begin{array}{l}\text { AC, antic } \\
\text { immuno } \\
\text { VV-ECM }\end{array}$ & $\begin{array}{l}\text { gulati } \\
\text { vulin; } \\
\text { venous }\end{array}$ & $\begin{array}{l}\text { CP, } \\
\text { PV, in }\end{array}$ & $\begin{array}{l}\text { ivaless } \\
\text { sive p } \\
\text { xtraco }\end{array}$ & $\begin{array}{l}\text { t plasr } \\
\text { itive pr } \\
\text { oreal m }\end{array}$ & $\begin{array}{l}\text {; F, fen } \\
\text { ure ve } \\
\text { nbrane }\end{array}$ & $\begin{array}{l}\text { le; GCs } \\
\text { tilation; } \\
\text { oxygen }\end{array}$ & $\begin{array}{l}\text { lucocorticoids; } \\
\text { PV/RTV, lopinav } \\
\text { on }\end{array}$ & $\begin{array}{l}\text { high-flow } \\
\text { havir; M, } \mathrm{m}\end{array}$ & $\begin{array}{l}\text { sal can } \\
\text {; NPPV }\end{array}$ & $\begin{array}{l}\text { la o } \\
\text { on-il }\end{array}$ & $\begin{array}{l}\text { gen } t \\
\text { asive }\end{array}$ & $\begin{array}{l}\text { erapy } \\
\text { ositi }\end{array}$ & $\begin{array}{l}\text { /IG, int } \\
\text { pressu }\end{array}$ & $\begin{array}{l}\text { avenous } \\
\text { e ventilation; }\end{array}$ \\
\hline
\end{tabular}

Ten patients (71.4\%) received lopinavir/ritonavir (LPV/RTV), six (42.9\%) received arbidol, eight (57.1\%) received at least one dose of convalescent plasma, $12(85.7 \%)$ received systemic glucocorticoids, and all patients received unfractionated heparin or low molecular weight heparin for anticoagulation. Nine (52.9\%) critical patients were discharged by April 62020.

We carried out bronchoscopy regularly, and bedside bronchoscopy examinations were performed in four patients (cases $8,9,10$, and 11$)$. We sent bronchoalveolar lavage fluid (BALF) for SARS-CoV-2 RNA testing and bacterial/fungal culture. Carbapenem-resistant $A$. baumannii (cases 8 and 11), carbapenem-resistant Klebsiella pneumoniae (cases 9, 10,11), and carbapenem-resistant Pseudomonas aeruginosa (cases 10 and 11 ) were detected in BALF. Antibacterial treatment was adjusted according to the results of drug sensitivity tests. Three patients (cases 8,10 , and 11$)$ also received continuous renal replacement therapy due to acute kidney injury.

Fifteen air samples were collected during high-risk procedures (HFNC, nebulisation, NPPV and tracheostomy). All samples tested negative for SARSCoV-2 RNA. One hundred and twenty-eight environment surface swabs from the isolation ward were also tested. One gastric tube swab and one anal tube swab tested positive for SARS-CoV-2. All oropharyngeal swabs taken from medical staff tested negative for SARS-CoV-2.

\section{Discussion}

Our centre received 14 critically ill patients with COVID-19 in 63 days. Nine (64.3\%) of these patients were discharged and two of three patients who received ECMO were able to withdraw successfully. We believe that these outcomes demonstrated the benefit of Individualized comprehensive treatment, reliable personal protection, and teamwork.

No antiviral treatment has proven effective for SARS-CoV-2. Potentially effective treatments include LPV/RTV, arbidol, remdesivir and convalescent plasma. Only LPV/RTV has been tested in a randomised clinical trial and was found to be no more effective than standard care(5). Although $68 \%$ of 61 patients who received remdesivir on a compassionate basis showed clinical improvement(6), the efficacy of this agent has yet to be confirmed in a randomised controlled trial. Moreover, remdesivir is still not universally accessible. Most of the patients admitted to our centre received antiviral drugs, 
but it is not known whether these agents had any benefit. To our knowledge, antiviral drugs should be administered in the early phase of influenzarelated pneumonia $(7,8)$. Antiviral treatment is not a magic bullet for critical viral infections, including influenza and Ebola virus(9-11). Clinicians should consider Individualized comprehensive treatment, given the absence of effective antiviral drugs.

Glucocorticoids are rapid-acting and have a strong anti-inflammatory effect. Coronavirus infection can cause a pathological inflammation reaction, leading to apoptosis of lymphocytes, infiltration of inflammatory cells, and a cytokine storm, which causes increased capillary permeability, alveolar exudates, and diffuse alveolar damage(12). The cytokine storm and replication of the virus plays an important role in development of disease. Levels of multiple cytokines are significantly increased in patients with COVID-19(13). Monocyte-macrophage infiltration and acute lung injury were also reported in an autopsy study of COVID-19(14,15). Organising pneumonia, which has distinctive radiological features and can be easily recognised by a chest physician(16), often responds to glucocorticoid therapy and can be secondary to viral pneumonia. Theoretically, glucocorticoids can be used for excessive inflammation or secondary organising pneumonia in COVID-19. However, high doses of glucocorticoids can lead to secondary infection, delayed viral clearance, and osteoporosis(17-19). There are reports showing that glucocorticoids were used in 18.6-44.9\% of hospitalised COVID-19 patients and at a higher rate in severe cases than in non-severe cases $(44.5-72.2 \%$ vs $11.0-35.3 \%)(2,13,20,21)$. However, use of glucocorticoids in COVID-19 or SARS-CoV-1 infection is still controversial. We think systemic glucocorticoids should not be used in all patients with COVID-19. However, we believe that clinicians can consider systemic glucocorticoids in patients with severe progressive disease, significantly increased inflammatory biomarker and cytokine levels, and signs of presumed secondary organising pneumonia on a CT scan of the lungs. Regimen of glucocorticoids was not definite, and we often use methylprednisolone $40-120 \mathrm{mg}$ for 10-14 days or longer. The lymphocyte count should be monitored when glucocorticoids are used. For patients with a lymphocyte count lower than $200 / \mathrm{mm}^{3}$, glucocorticoids should be used with caution. Two patients who received glucocorticoids are shown in Fig. 1. The risk-benefit ratio of glucocorticoids in COVID-19 should be further assessed in randomised controlled trials.

Convalescent plasma is passive administration of antibodies collected from individuals who have recovered from an infectious illness and has been used in patients who are critically ill with other viral infections $(22,23)$. Several studies in small numbers of patients with COVID-19 showed that convalescent plasma can be beneficial in terms of radiological resolution and a decreased viral load and has a survival benefit(24-26). The US Food and Drug Administration has approved its use for critical COVID-19 cases(27). Since the duration of efficacy of the antibodies is not known, no convalescent plasma regimen has been defined. Based on our experience in patients with SARS-CoV- 1 infection, we used convalescent plasma on one or more occasions at a dosage of $3-5 \mathrm{ml} / \mathrm{kg}$ with a receptor binding domain antibody titre of $>1: 160$ according to the clinical response and the results of viral tests(28). Seven of the eight patients who received convalescent plasma at our centre showed clinical improvement and four of these patients were discharged to home. Given that all patients received comprehensive treatment, it was difficult to evaluate the efficacy of convalescent plasma in this study, and further evaluation is needed in randomised controlled trials.

Coagulopathy is common and D-dimer is an independent risk factor for death in patients with COVID-19(29). Hyaline thrombi were found in the pulmonary microvessels in an autopsy study of COVID-19(30). Whether the pulmonary circulation and the right ventricular function affect the prognosis has not been studied as yet in patients with COVID-19. We suggest prophylactic anticoagulation in all patients without contraindications.

Respiratory support techniques, including HFNC, NPPV, IPPV, and ECMO, are important in the treatment of viral pneumonia and acute respiratory distress syndrome. HFNC and NPPV may increase the risk of aerosol dissemination and cause a delay in use of appropriate support devices. Since we did not detect SRAS-CoV-2 in air samples or in most of the environmental surface swabs, we think it is safe to carry out some high-risk procedures, including bronchoscopy and tracheostomy, with appropriate personal protection. We also took extra protective measures during high-risk procedures (Table 3), including placing surgical masks on patients receiving HFNC and N95 masks around the endotracheal tube during tracheostomy (Fig. 2). Three patients died before we can carry out intubation and mechanical ventilation and we presume that the cause of death was delayed intubation. We suggest that patients should be monitored for disease progression, especially respiratory rate and oxygenation. When the respiratory rate increases significantly (e.g. to 30 per minute), intubation and IPPV should be implemented early to avoid multiple organ dysfunction due to prolonged hypoxemia. 
Table 3

Additional protective measures during high-risk procedures

\begin{tabular}{|ll|}
\hline Procedures & Extra protective measure \\
\hline Taking nasopharyngeal swab & Ordinary head cover \\
\hline Bronchoscopy & Positive pressure head cover \\
\hline Tracheostomy & Positive pressure head cover \\
& Positive pressure head cover and \\
\hline HFNC & N95 masks around the endotracheal tube \\
\hline NIV & Surgical masks for patients \\
\hline IPPV & Ordinary head cover and \\
& avoidance in front of the exhalation valve \\
\hline $\begin{array}{l}\text { Abbreviations: HFNC, high-flow nasal cannula oxygen therapy; IPPV, invasive positive pressure ventilation; NPPV, non-invasive positive pressure } \\
\text { ventilation }\end{array}$ & $\begin{array}{l}\text { Adding exhalation filter and } \\
\text { closed suction }\end{array}$ \\
\hline
\end{tabular}

ECMO is an important therapy for severe respiratory failure and can gain the time needed for treatment of underlying disease and repair of lung. It has been reported to save lives in patients with severe influenza pneumonia(31). According to our experience of ECMO in three patients with COVID-19, lung injury was severe in critical cases and lung fibrosis developed in the late phase. Therefore, the duration of mechanical ventilation and ECMO may be very long, leading to a high risk of ventilation-induced lung injury and nosocomial infection. All three patients who received ECMO at our centre developed ventilator-related pneumonia (two had ventilator-related pneumonia before transferred to our centre) and one developed a catheter-related bloodstream infection and died. This suggests that it is important to deal with complications in the treatment of critical COVID-19 cases.

Tracheostomy was performed in five patients when the endotracheal tube could not be extubated early to reduce sedation and keep them alert and able to cough. We also implemented closed suction, bronchoscopy as needed, and strict hand hygiene to control ventilator-related pneumonia. We used a peripherally inserted central catheter instead of a central venous catheter to reduce the risk of catheter-related bloodstream infection. ECMO is a complicated procedure and should be performed only by an experienced team. We suggest that patients with critical COVID-19 should be transferred to an experienced centre(32).

Doctors and nurses must work while wearing protective equipment in the isolation ward for lengthy periods, which requires considerable physical strength. Therefore, realistic shift plans and suitable staff are very important. Treatment of patients with critical COVID-19 is complicated and entails use of various respiratory techniques. A team approach must be implemented. Our medical team was led by PCCM doctors. All our fellows had received PCCM fellowship and had been on-call for at least 6 months in the medical ICU as chief residents, which is important in the treatment of patients with critical COVID-19.

This study has limitations. Only 14 critically ill COVID-19 patients from a single centre were included. It would be better to included more patients from more centres, and even in other countries. However, the data in our study provide an early reference for the management of critical COVID-19 patients, especially when we do not have effective therapies.

\section{Conclusions}

In conclusion, a team that includes highly qualified and dedicated professionals is paramount in the treatment of patients with severe COVID-19 infection. Comprehensive Individualized treatment, appropriate personal protective equipment, and teamwork may improve the prognosis in patients who become critically ill with this disease.

\section{List Of Abbreviations}

BALF: bronchoalveolar lavage fluid

COVID-19: Coronavirus disease 2019

ECMO: extracorporeal membrane oxygenation

$\mathrm{FiO}_{2}$ : fraction of inspired oxygen

HFNC: high-flow nasal cannula

ICU: intensive care unit 
IPPV: invasive positive pressure ventilation

LPV/RTV: lopinavir/ritonavir

NPPV: non-invasive positive pressure ventilation

PCCM: pulmonary and critical care medicine

SARS-CoV-2: severe acute respiratory syndrome coronavirus 2

\section{Declarations}

\section{Ethics approval and consent to participate}

The study was independently approved by the ethics committees of the abovementioned hospital (2020-21-K16), and written informed consent was waived due to the retrospective nature and urgent need to collect data.

\section{Consent for publication}

Written informed consent for publication of the image of the patient has been collected.

\section{Availability of data and materials}

The datasets used and/or analysed during the current study are available from the corresponding author on reasonable request.

\section{Competing interests}

The authors declare that they have no competing interests.

\section{Funding}

This work was supported by Zhejiang University Special Scientific Research Fund for COVID-19 Prevention and Control [grant number 2020XGZX008]; CAMS Innovation Fund for Medical Sciences [grant number 2018-I2M-1-003]; and Non-profit Central Research Institute Fund of CAMS [grant number 2019TX320006]. The funding body took no role in the design of the study and collection, analysis, and interpretation of data and in writing the manuscript.

\section{Authors' contributions}

All authors made substantial contributions to the conception and design of the study, data acquisition, review and approval of the final manuscript. Drs. KH, ZZ, ML, YF, YY, YC, JX, JD, and QZ performed experiments. Drs. KH, ZZ, JD and QZ analyzed data. Drs. YC, JD and QZ were responsible for the drafting of the manuscript.

\section{Acknowledgements}

We thank Tongji Hospital and Tongji Medical College for their great support to this work.

\section{References}

1. Guan WJ, Ni ZY, Hu Y, et al. Clinical characteristics of coronavirus disease 2019 in China. N Engl J Med. 2020; doi: 10.1056/NEJMoa2002032.

2. Yang $X, Y u Y, X u ~ J$, et al. Clinical course and outcomes of critically ill patients with SARS-CoV-2 pneumonia in Wuhan, China: a single-centered, retrospective, observational stuy. Lancet Respir Med. 2020; doi: 10.1016/S2213-2600(20)30079-5.

3. Coronavirus disease (COVID-19) situation dashboard. https://www.who.int/emergencies/diseases/novel-coronavirus-2019. Accessed April 11, 2020.

4. Poston JT, Patel BK, Davis AM. Management of critically ill adults with COVID-19. JAMA. 2020; doi: 10.1001/jama.2020.4914.

5. Cao B, Wang Y, Wen D, et al. A trial of lopinavir-ritonavir in adults hospitalized with severe Covid-19. N Engl J Med. 2020; doi:10.1056/NEJMoa2001282.

6. Grein J, Ohmagari N, Shin D, et al.Compassionate use of remdesivir for patients with severe Covid-19. N Engl J Med. 2020; doi:10.1056/NEJMoa2007016.

7. Katzen J, Kohn R, Houk JL, et al. Early oseltamivir after hospital admission is associated with shortened hospitalization: a 5-year analysis of oseltamivir timing and clinical outcomes. Clin Infect Dis. 2019;69:52-58.

8. Muthuri SG, Venkatesan S, Myles PR, et al. Effectiveness of neuraminidase inhibitors in reducing mortality in patients admitted to hospital with influenza A H1N1pdm09 virus infection: a meta-analysis of individual participant data. Lancet Respir Med. 2014;2:395-404. 
9. Marty FM, Vidal-Puigserver J, Clark C,et al. Intravenous zanamivir or oral oseltamivir for hospitalised patients with influenza: an international, randomised, double-blind, double-dummy, phase 3 trial. Lancet Respir Med. 2017;5:135-146.

10. South East Asia Infectious Disease Clinical Research Network. Effect of double dose oseltamivir on clinical and virological outcomes in children and adults admitted to hospital with severe influenza: double blind randomised controlled trial. BMJ. 2013;346:f3039.

11. Mulangu S, Dodd LE, Davey RT, et al. A randomized, controlled trial of Ebola virus disease therapeutics. N Engl J Med. 2019;381:2293-2303.

12. Channappanavar R, Perlman S. Pathogenic human coronavirus infections: causes and consequences of cytokine storm and immunopathology. Semin Immunopathol. 2017;39:529-539.

13. Huang C, Wang Y, Li X, et al. Clinical features of patients infected with 2019 novel coronavirus in Wuhan, China. Lancet. 2020;395:497-506.

14. Xu Z, Shi L, Wang Y, et al. Pathological findings of COVID-19 associated with acute respiratory distress syndrome. Lancet Respir Med. 2020;8:420422.

15. Tian S, Hu W, Niu L, et al. Pulmonary pathology of early-phase 2019 novel coronavirus (COVID-19) pneumonia in two patients with lung cancer. J Thorac Oncol. 2020;8:420-422.

16. Roberton BJ, Hansell DM. Organizing pneumonia: a kaleidoscope of concepts and morphologies. Eur Radiol. 2011;21:2244-2254.

17. Wang H, Ding Y, Li X, et al. Fatal aspergillosis in a patient with SARS who was treated with corticosteroids. N Engl J Med. 2003;349:507-508.

18. Lee N, Allen Chan KC, Hui DS, et al. Effects of early corticosteroid treatment on plasma SARS-associated coronavirus RNA concentrations in adult patients. J Clin Virol. 2004;31:304-309.

19. Zhao R, Wang H, Wang X, et al. Steroid therapy and the risk of osteonecrosis in SARS patients: a dose-response meta-analysis. Osteoporos Int. 2017;28:1027-1034.

20. Wang D, Hu B, Hu C, et al. Clinical characteristics of 138 hospitalized patients with 2019 novel coronavirus-infected pneumonia in Wuhan, China. JAMA. 2020; doi:10.1001/jama.2020.1585.

21. Chen N, Zhou M, Dong X, et al. Epidemiological and clinical characteristics of 99 cases of 2019 novel coronavirus pneumonia in Wuhan, China: a descriptive study. Lancet. 2020;395:507-513.

22. Mair-Jenkins J, Saavedra-Campos M, Baillie JK, et al. The effectiveness of convalescent plasma and hyperimmune immunoglobulin for the treatment of severe acute respiratory infections of viral etiology: a systematic review and exploratory meta-analysis. J Infect Dis. 2015;211:80-90.

23. van Griensven J, Edwards T, de Lamballerie X, et al. Evaluation of convalescent plasma for Ebola virus disease in Guinea. N Engl J Med. 2016;374:33-42.

24. Shen C, Wang Z, Zhao F, et al. Treatment of 5 critically ill patients with COVID-19 with convalescent plasma. JAMA. 2020; doi:10.1001/jama.2020.4783.

25. Zhang B, Liu S, Tan T, et al. Treatment with convalescent plasma for critically ill patients with SARS-CoV-2 infection. Chest. 2020; doi:10.1016/j.chest.2020.03.039.

26. Duan K, Liu B, Li C, et al. Effectiveness of convalescent plasma therapy in severe COVID-19 patients. Proc Natl Acad Sci U S A. 2020; doi:10.1073/pnas.2004168117.

27. Tanne JH. Covid-19: FDA approves use of convalescent plasma to treat critically ill patients. BMJ. 2020; 368 : $\mathrm{m} 1256$.

28. Bloch EM, Shoham S, Casadevall A, et al. Deployment of convalescent plasma for the prevention and treatment of COVID-19. J Clin Invest. 2020; doi:10.1172/JCl138745.

29. Zhou F, Yu T, Du R, et al. Clinical course and risk factors for mortality of adult inpatients with COVID-19 in Wuhan, China: a retrospective cohort study. Lancet. 2020;395:1054-1062.

30. Yao XH, Li TY, He ZC, et al. A pathological report of three COVID-19 cases by minimally invasive autopsies. Zhonghua Bing Li Xue Za Zhi. 2020;49:E009; doi: 10.3760/cma.j.cn112151-20200312-00193

31. Australia and New Zealand Extracorporeal Membrane Oxygenation (ANZ ECMO) Influenza Investigators1, Davies A, Jones D, Bailey M, et al. Extracorporeal membrane oxygenation for 2009 Influenza A (H1N1) acute respiratory distress syndrome. JAMA. 2009;302:1888-1895.

32. Chen M, Evans A, Gutsche J. Success With VV ECMO for respiratory failure: is it the device, the center, or both? J Cardiothorac Vasc Anesth. 2018;32:1160-1161.

\section{Figures}




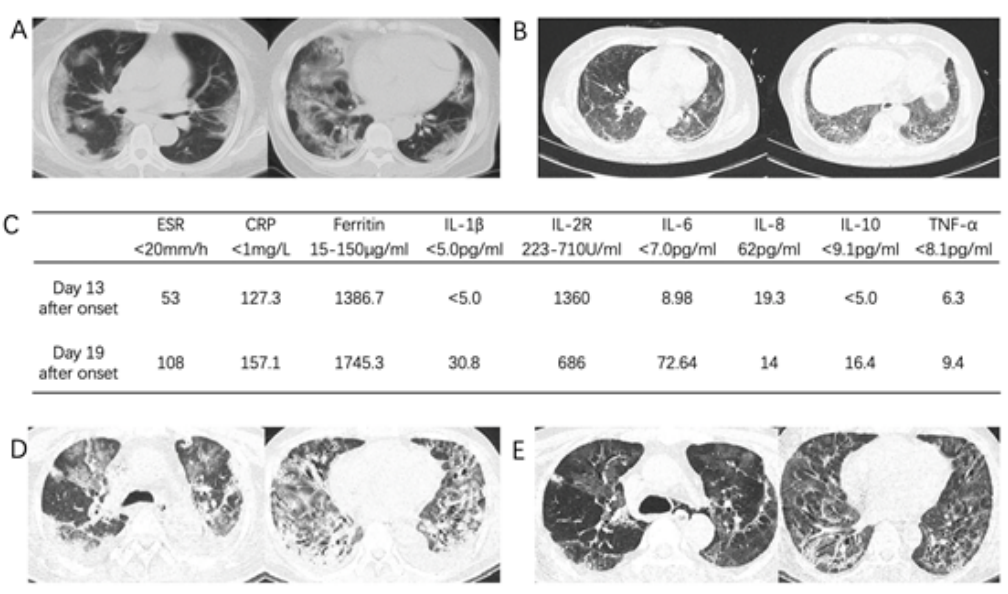

Figure 1

Radiological changes in two patients treated with glucocorticoids. (a-c) Patient 1: A 60-year-old woman with diabetes who was hospitalised because of a 12-day history of fever, cough, and dyspnoea and a positive nasopharyngeal swab test for SARS-CoV-2 RNA. A chest computed tomography (CT) scan showed bilateral opacity on day 7 after onset of symptoms (a). Despite high-flow nasal cannula oxygen therapy and administration of lopinavir/ritonavir, her respiratory function worsened with a respiratory rate 35-40 per minute. Intubation and invasive positive pressure ventilation were initiated. Laboratory tests revealed significantly higher levels of multiple inflammatory factors on day 19 after onset than on day 13 (c). Her lymphocyte count was $1310 / \mathrm{mm} 3$. Intravenous methylprednisolone (80 mg per day) was initiated. Her respiratory function recovered and she was extubated 5 days later. The CT scan before she was discharged on day 28 after onset showed that most of the opacity had resolved (b). (d-e) Patient 10: A 42-year-old man with no underlying disease who could not be weaned from mechanical ventilation and extracorporeal membrane oxygenation (ECMO) by day 42 after onset of COVID-19. A chest CT scan showed presumed organising pneumonia (d). Intravenous methylprednisolone (40 $\mathrm{mg} /$ day) was administered. His respiratory function improved and he was finally weaned from mechanical ventilation and ECMO. A chest CT scan obtained 10 days later demonstrated absorption of the opacity.
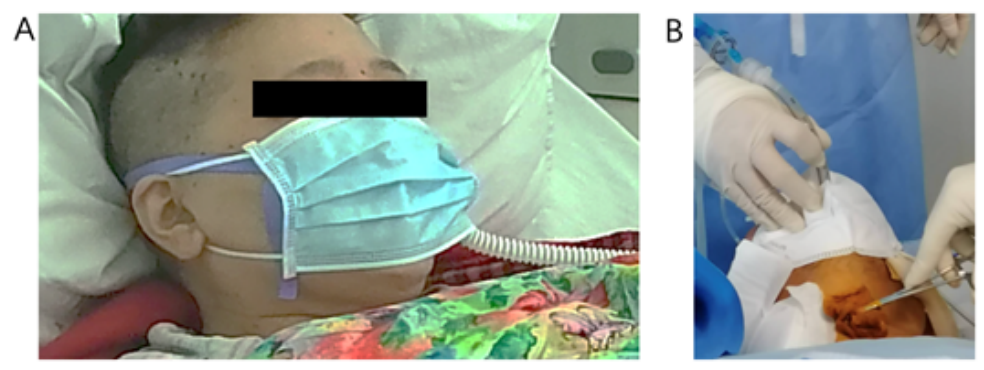

\section{Figure 2}

Measures used to reduce aerosol exposure at our centre. (a) Use of a surgical mask during high-flow nasal cannula oxygen therapy. (b) Placement of an N95 mask around the endotracheal tube before its removal during tracheostomy.

\section{Supplementary Files}

This is a list of supplementary files associated with this preprint. Click to download.

- Additionalfile1.pdf

- Additionalfile1.pdf 